SUBJECT AREAS:

BIOMATERIALS

POLYMER SYNTHESIS

POLYMERS

Received

6 August 2013

Accepted

27 February 2014

Published

17 March 2014

Correspondence and requests for materials should be addressed to C.G. (chaogao@zju. edu.cn)

* These authors contributed equally to this work.

\section{Topological Polymers by Step-growth Thiol-yne Approach}

\author{
Jin Han ${ }^{1 *}$, Yaochen Zheng ${ }^{1,2 *}$, Bo Zhao', Sipei Li', Yuanchao Zhang' \& Chao Gao'
}

${ }^{1}$ MOE Key Laboratory of Macromolecular Synthesis and Functionalization, Department of Polymer Science and Engineering, Zhejiang University, 38 Zheda Road, Hangzhou 310027, P. R. China, ${ }^{2}$ College of Chemistry and Chemical Engineering, Yantai University, 30 Qingquan Road, Yantai 264005, P. R. China.

Sequence-controlled polymers (SCPs) such as DNA and proteins play an important role in biology. Many efforts have been devoted to synthesize SCPs in the past half a century. However, to our knowledge, the artificial sequences containing independently functional groups have never been reported. Here, we present a facile and scalable approach based on radical-initiated step-growth polymerization to synthesize sequence-controlled functional polymers (SCFPs) with various topologies, covering from linear to random and hyperbranched polymers. The functional groups, such as $\mathrm{OH} / \mathrm{NH}_{2}, \mathrm{OH} / \mathrm{COOH}$, and $\mathrm{NH}_{2} / \mathrm{N}_{3}$, alternately arranged along the chain, which were further selectively functionalized to achieve DNA-mimic and hetero-multifunctional SCPs. This user-friendly strategy exhibits advantages of commercially available monomers, catalyst-free process, fast reaction, high yield and water solvent, opening a general approach to facile and scalable synthesis of SCFPs.

equence-controlled polymers (SCPs) represent a kind of macromolecules composed of orderly arranged monomer units of different chemical nature ${ }^{1}$. SCPs are crucial in biological functions. For instance, the genetic code is stored in the sequence of bases and instructs the processes of DNA replication, transcription, and translation. To achieve such advanced functions, both the sequentially repeated units and the self-recognizing functional groups are the crucial prerequisites. Accordingly, various strategies have been developed to synthesize SCPs since $1963^{1-5}$ including, 1) the most extensively applied condensation polymerization between two bifunctional symmetric or nonsymmetric monomers $\left.{ }^{6}, 2\right)$ templating polymerization aided by complementary macromolecule templates or joint monomers templates recently innovated by Sawamoto et al. ${ }^{7}, 3$ ) step-by-step increment such as the famous Merrifield solid-phase synthesis ${ }^{8}$, and a recently developed liquid-phase synthesis employing soluble polymer support, and a special activation/addition process $\left.{ }^{9}, 4\right)$ kinetic control in chain cross propagation (i.e., typical copolymerization of styrene and maleic anhydride) ${ }^{10,11}$, 5) polymerization of short sequence-defined oligomers to produce periodic copolymers ${ }^{12}$, and 6) quantitative and regioselective insertion of monomer into polymer chains ${ }^{13,14}$. However, all of the artificial SCPs have no independent functional groups suspended on backbone $e^{6-30}$, and thus it is quite difficulty to further design and introduce specific functional moieties on those SCPs. Therefore, to readily and efficiently synthesize sequence-controlled functional polymers (SCFPs) is still a big challenge. Moreover, other topological SCFPs such as sequential dendritic polymers have never been reported yet, although three-dimensional biomacromolecules widely exist in nature.

Here we present a facile and general strategy to synthesize SCFPs with tailored hetero-functional groups, such as $\mathrm{OH} / \mathrm{NH}_{2}, \mathrm{OH} / \mathrm{COOH}$, and $\mathrm{NH}_{2} / \mathrm{N}_{3}$, via radical-initiated step-growth (RISG) polymerization in water. The whole process is catalyst-free and the monomers are commercially available. The SCFPs with independently hetero-functional groups are tolerant to further selective modification. This methodology opens the avenue to scalable synthesis of DNA-mimetic sequential polymers in a fast, concise, precise and reliable manner.

\section{Results}

Strategy design. We designed RISG polymerization approach on the basis of thiol-yne click chemistry to achieve SCFPs, which combines the advantages of radical polymerization (e.g., tolerance to functional groups) and condensation polymerization (e.g., regular sequence units). Figure 1a shows the polymerization protocol and mechanism. At the Initiation step, under UV-irradiation or heating, the initiators such as 2,2-dimethoxy-2phenylacetophenone (DMPA) or 4,4'-azobis(4-cyanovaleric acid) (ACVA) decompose into two primary 
a

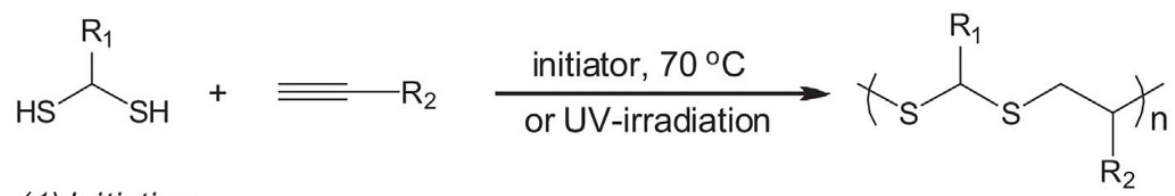

(1) Initiation<smiles>[R17][14CH]I</smiles>

(2) Propagation

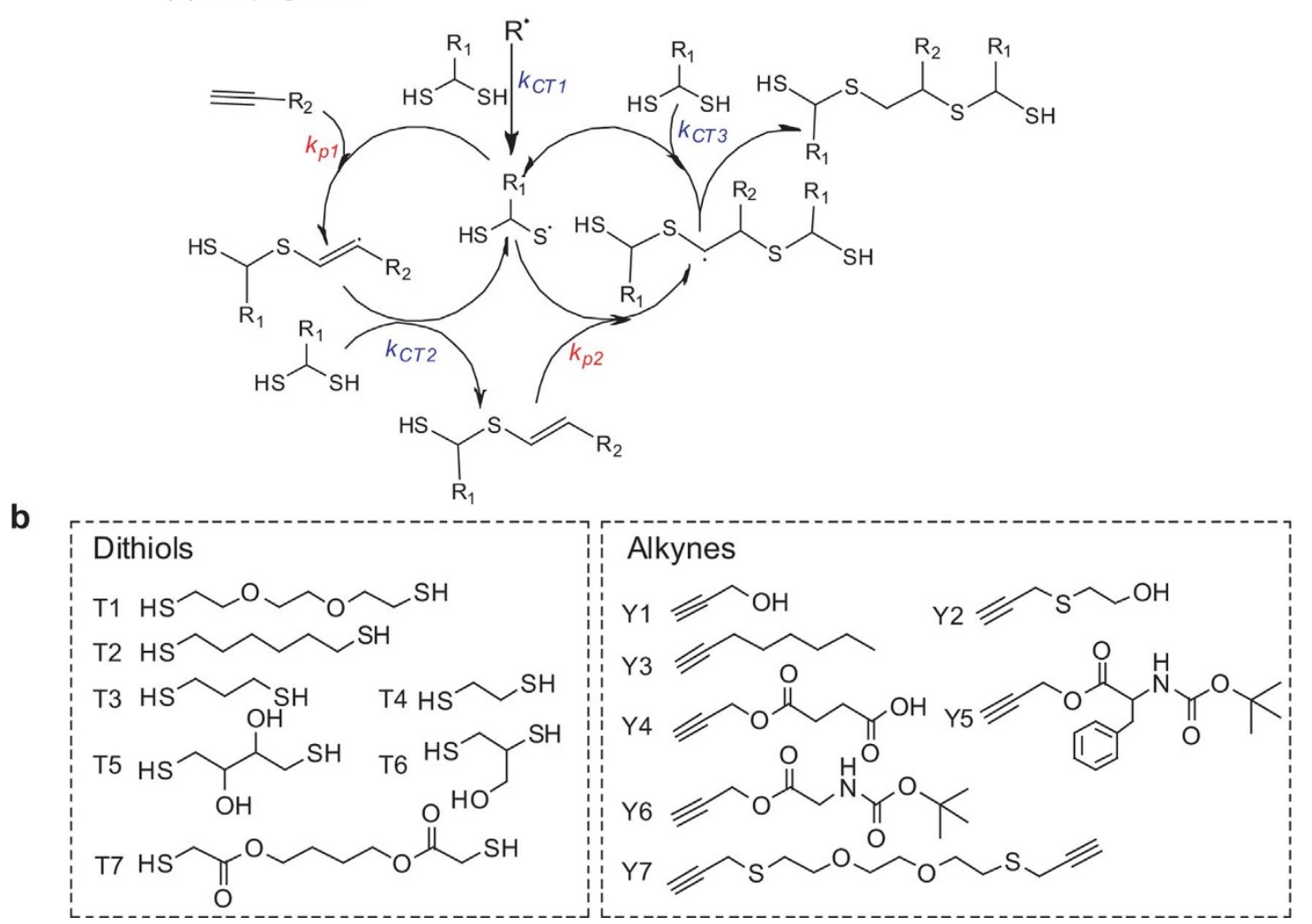

Figure 1 | Radical-initiated step-growth polymerization (RISG) of dithiols and alkynes. (a), RISG polymerization mechanism by alternative radical addition and hydrogen abstraction reaction. (b), employed monomers of dithiols and alkynes.

radicals $\left(k_{\mathrm{d}}\right)$, which take a hydrogen atom from a thiol group to form a thiyl radical $\left(k_{C T 1}\right)$. Then the step-by-step Propagation begins: the addition of thiyl radical to an alkyne results in carbon-carbon double bond radical $\left(k_{p 1}\right)$, which subsequently abstracts a hydrogen atom from another thiol group to generate a vinyl sulfide moiety and another thiyl radical $\left(k_{\mathrm{CT} 2}\right)$; the following addition of vinyl sulfide to a thiyl radical to give birth to carbon-carbon single bond radical with a unit of S-R1-S-R2 $\left(k_{\mathrm{p} 2}\right)$ (herein $\mathrm{S}$ denotes the functional thioether bond since it can be further modified into sulfone unit, and R1 and R2 represent functional groups of dithiol and alkyne, respectively); repeating of the alternative hydrogen-abstraction and radical-addition reactions offers targeted SCFPs with regular sequence of S-R1-S-R2 (Figure S1).

In the propagation, the chain transfer constant of thiol groups $(>48)$ is big enough to quench the carbon-centered radicals ${ }^{31}$, ensuring controlled step-by-step growth for the synthesis of SCPs. Significantly, the independent functional groups (e.g., S, R1 and R2) remain intact during the RISG polymerization because the reactive rate of radical to the thiol group is considerably higher than that of radical to the functional groups. Therefore, our RISG polymerization strategy enables sequential unit of backbone and regularly arranged functional groups simultaneously, achieving high molecular weight of SCFPs in the absence of any metal catalysts. Since dithiols and alkynes are widely available and R1 and R2 can be tailor-made, various SCFPs are easily accessible through the general RISG polymerization approach.

S-R-S-R2-type linear SCFPs. As shown in Figure 1b, series of dithiols and alkynes are selected to synthesize linear SCFPs with SR-S-R2 sequences. At first, the common monomers of T1 and Y1 were employed to verify our RISG polymerization strategy. To mimic the synthesis surrounding of biomacromolecules, we chose water as the reaction solvent. By initiating with water-soluble ACVA, the RISG polymerization of $\mathrm{T} 1$ and $\mathrm{Y} 1$ produced an S-R-S-OH-type SCFP (T1-Y1) with a weight-average molecular weight $\left(M_{\mathrm{n}}\right)$ of 5 $900 \mathrm{~g} \mathrm{~mol}^{-1}$ and polydispersity index (PDI) of 1.80 in an ultrahigh yield (95\%) (Table S1). The chemical structure of T1-Y1 was confirmed by ${ }^{1} \mathrm{H}$ NMR and ${ }^{13} \mathrm{C}$ NMR spectroscopy (Figure S1). No signals of vinyl groups were found in the ${ }^{1} \mathrm{H}$ NMR spectrum of T1Y1, implying the thiol-yne based RISG polymerization was proceeding apace. Thus, high molecular weight of SCFP with weight-average degree of polymerization $\left(D P_{\mathrm{w}}\right)$ of 44.6 was obtained. Such a value of repeating sequence is obviously higher than those of previous sequential polymers $\left(D P_{\mathrm{w}} 7.0-39.4\right)^{11,12,32,33}$ synthesized via radical-initiated polymerizations (Table S2).

To fully understand the RISG polymerization, different solvents were tried as the reaction medium. As a result, $M_{\mathrm{n}}$ of T1-Y1 showed 

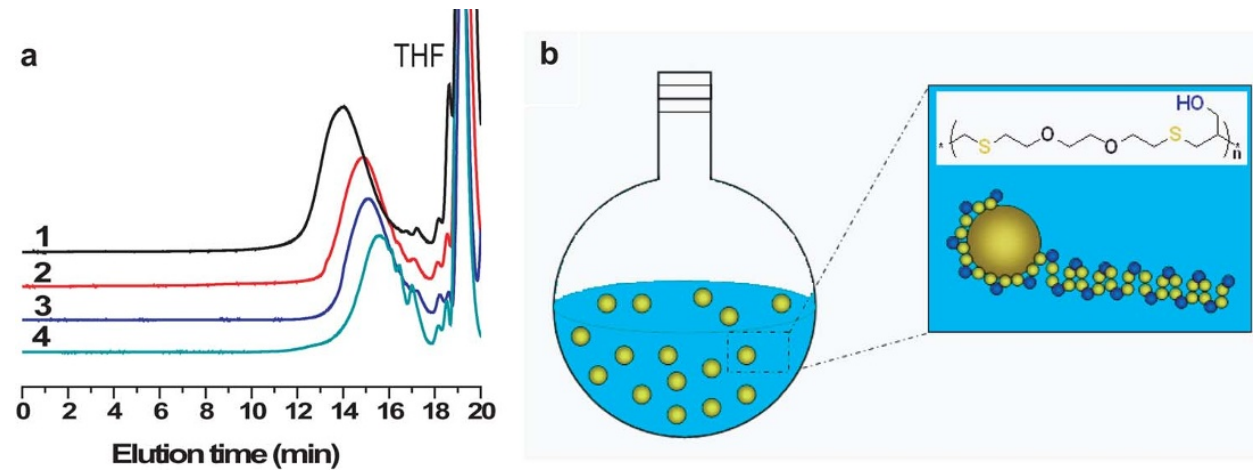

Figure 2 Typical GPC curves of SCFPs and interfacial polymerization model. (a), GPC curves of T1-Y1 polymerized in water (1), toluene (2), DMF (3), and bulk (4), respectively. (b), Interfacial reaction mechanism for the polymerization of T1 and Y1.

an order of $\mathrm{H}_{2} \mathrm{O}\left(5900 \mathrm{~g} \mathrm{~mol}^{-1}\right)>$ toluene $\left(4000 \mathrm{~g} \mathrm{~mol}^{-1}\right)>\mathrm{DMF}$ (1 $\left.900 \mathrm{~g} \mathrm{~mol}^{-1}\right) \approx$ bulk (1 $900 \mathrm{~g} \mathrm{~mol}^{-1}$ ), as confirmed by gel permeation chromatography (GPC) measurements (Figure 2a). Except performing reactions in bulk, GPC curves of T1-Y1s were unimodal, implying the side reactions involved in RISG polymerization were largely avoided in water and organic solvents. Thus, water is the best reaction medium for the thiol-yne polymerizations. Since one monomer (Y1) is soluble in water, and another one (T1) is insoluble in water, the mode of interfacial polymerization is proposed to illustrate the reaction process (Figuer $2 \mathrm{~b}$ ). Under heating, the water soluble initiator of ACVA cracks into primary radicals which diffuse onto the surface of T1 oil-droplets and attack thiol groups to form

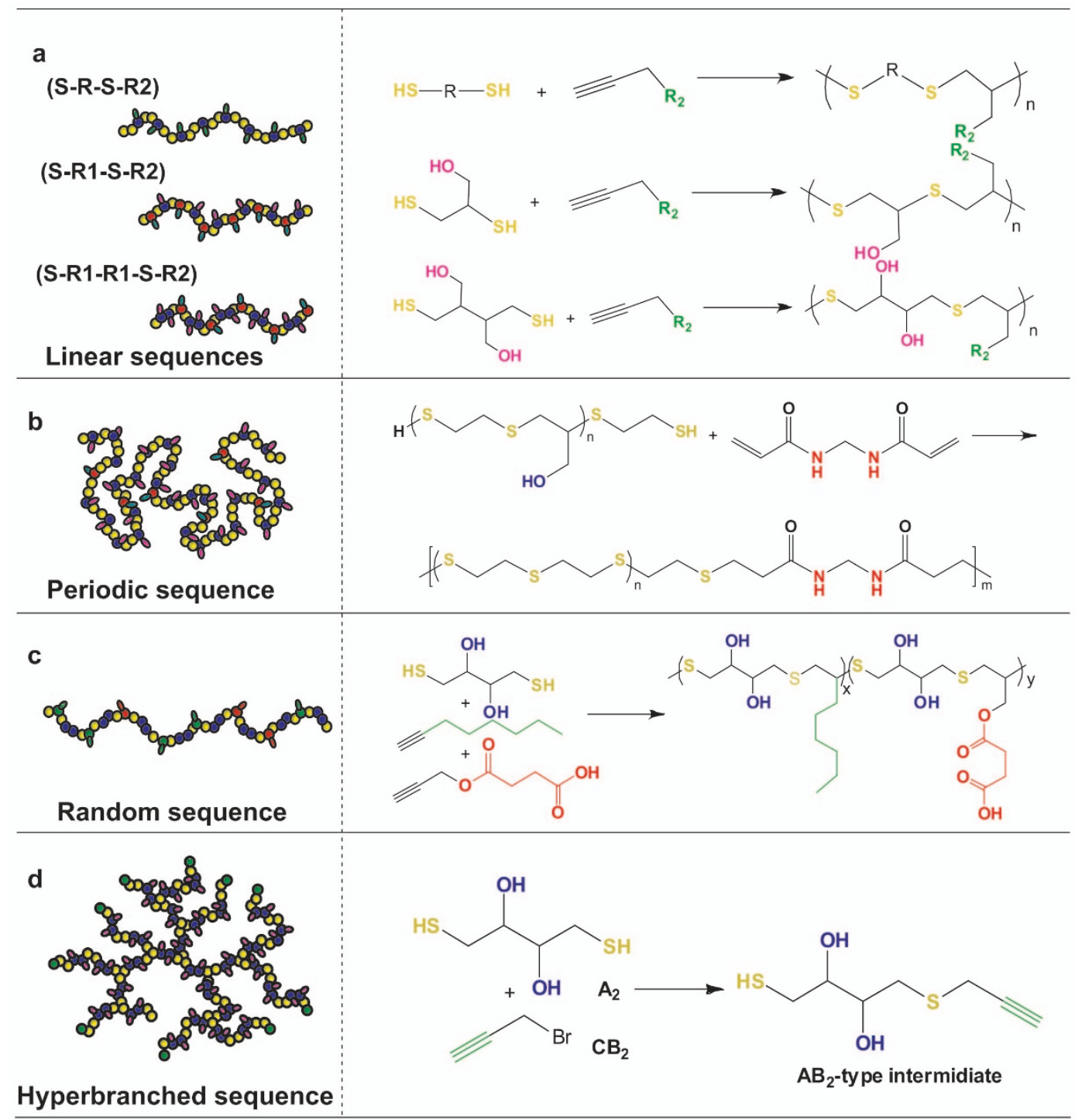

Figure 3 Sequence-controlled hetero-functional, topological polymers. R1 and $\mathbf{R} 2$ denote two different functional groups and $\mathbf{S}$ represents thioether bond which can be transformed into sulfone bond by oxidation. 


\begin{tabular}{|c|c|c|c|c|}
\hline Topological SCFP $a$ & $M_{\mathrm{n}}\left(\mathrm{g} \mathrm{mol}^{-1}\right)$ & $D P_{\mathrm{n}}$ & PDI & Yields (\%) \\
\hline Linear SCFP (T 1-Y 1) & 5900 & 44.6 & 1.80 & 95.1 \\
\hline Linear SCFP (T3-Y1) & 4000 & 42.1 & 1.74 & 96.3 \\
\hline Linear SCFP (T4-Y1) & 3600 & 38.7 & 1.61 & 98.7 \\
\hline Linear SCFP (T 1-Y2) & 3800 & 14.7 & 1.13 & 95.2 \\
\hline Linear SCFP (T 1-Y3) & 6600 & 32.2 & 1.41 & 98.0 \\
\hline Linear SCFP (T5-Y3) & 5100 & 33.7 & 1.75 & 97.1 \\
\hline Linear SCFP (T 1-Y4) & 5600 & 46.4 & 2.77 & 98.1 \\
\hline Linear SCFP (T5-Y4) & 4300 & 25.8 & 1.85 & 97.6 \\
\hline Linear SCFP (T2-Y5) & 3600 & 12.1 & 1.53 & 95.8 \\
\hline Linear SCFP (T5-Y5) & 3200 & 18.2 & 2.59 & 90.5 \\
\hline Linear SCFP (T4-Y6) & 4000 & 19.2 & 1.48 & 97.2 \\
\hline Linear SCFP (T5-Y6) & 2500 & 9.5 & 1.40 & 96.5 \\
\hline Linear SCFP (T6-Y6) & 2400 & 10.1 & 1.42 & 95.8 \\
\hline Periodic SCFP (T4-Y 1-BIS) & 7000 & 51.0 & 1.64 & 95.6 \\
\hline Random SCFP (T5-Y3-Y4) & 5700 & 31.9 & 1.60 & 96.3 \\
\hline Hyperbranched SCFP & 3800 & 25.0 & 1.27 & 82.6 \\
\hline
\end{tabular}

thiyl radicals. The thiyl radicals keep the activity in the T1 droplets until an alkyne molecule diffuses onto the surface of oil-droplets, and then the step-growth addition reaction takes place at the water/oil interface. The amphiphilic intermediates of oligomers and macroradicals covered $\mathrm{T} 1$ droplets like a surfactant, and thus had more chance than that in the cases of solution and bulk polymerizations to react with both thiyl radicals and Y1, resulting in the highest molecular weight of product.
To demonstrate the universality of our RISG polymerization methodology, we chose representative monomers of functional dithiols (e.g., T1-T4) and alkynes (e.g., Y1-Y4) to synthesize a series of S-R-S-R2-type SCFPs in water (Figures $1 \mathrm{~b}$ and $3 \mathrm{a}$ ). Their molecular weights and $D P_{\mathrm{n}} \mathrm{s}$ are listed in Table 1, and the corresponding structures were confirmed by NMR spectroscopy (Figures S2-S12). The results indicate that the RISG polymerization approach possesses the following advantages: 1 ) high yield (>90\%) and high efficiency (1-8 h of polymerization time), 2) relatively high molecular weight $\left(M_{\mathrm{n}}=3800-6600 \mathrm{~g} \mathrm{~mol}^{-1}\right)$, 3) availability of sequentially and variously functional groups (thioether/OH, thioether/COOH, and thioether/alkyl, etc.) for the resulting SCFPs.

Notably, for the monomers of thiols and alkynes that are both insoluble in water, the polymerization also proceeded quickly in water using 2, 2' -azobisisobutyronitrile (AIBN) as the initiator. For instance, the polymerization of T1 and Y3 produced the SCFP with $M_{\mathrm{n}}=6600 \mathrm{~g} \mathrm{~mol}^{-1}$ and PDI $=1.41$ (Table S3). In this case, the RISG polymerization took place according to the suspension polymerization mechanism.

Complex hetero-functional SCFPs. Generally, a method can only result in SCPs with the same sequence in previous reports owing to the limits of specific catalysts or polymerization mechanism ${ }^{9,12,13,29,30}$. So to achieve SCFPs with different sequences of structures and components through single approach is a challenge hitherto. Here we readily solved such a big problem by expending our RISG polymerization strategy to more functional monomers. Besides the S-R-S-R2-type SCFPs mentioned above, we synthesized S-R1-SR2-type SCFPs expediently by polymerization of T6 and alkynes<smiles>CC(C)(C)CSCC(CCSC(CO)CSC(C)(C)C)COC(=O)NCC(=O)OC(C)(C)C</smiles>

T6-Y6<smiles>CC(C)(C)OC(=O)CNC(=O)OCC(CCSC(COC(=O)CN)CSC(C)(C)C)C(C)(C)C</smiles>

$\mathrm{T}_{6} \mathrm{~N}_{3}-\mathrm{Y} 6$<smiles>CC(C)SCC(COC(=O)CN)SCC(COC(=O)CN)CC(C)(C)C</smiles>

$\mathrm{T} 6 \mathrm{~N}_{3}-\mathrm{Y} 6 \mathrm{NH}_{2}$<smiles>Cc1cn(CCC(=O)OCc2cn(CC(=O)OCC(CSC(C)(C)C)SCCCCCC(=O)CN)nn2)c(=O)[nH]c1=O</smiles>

T6Thy-Y6NH 2<smiles>Cc1cn(CCC(=O)OCc2cn(CC(=O)OCC(S)CSC(C)(C)C)nn2)c(=O)[nH]c1=O</smiles>

T6Thy- $\mathrm{Y}_{6} \mathrm{~N}_{3}$

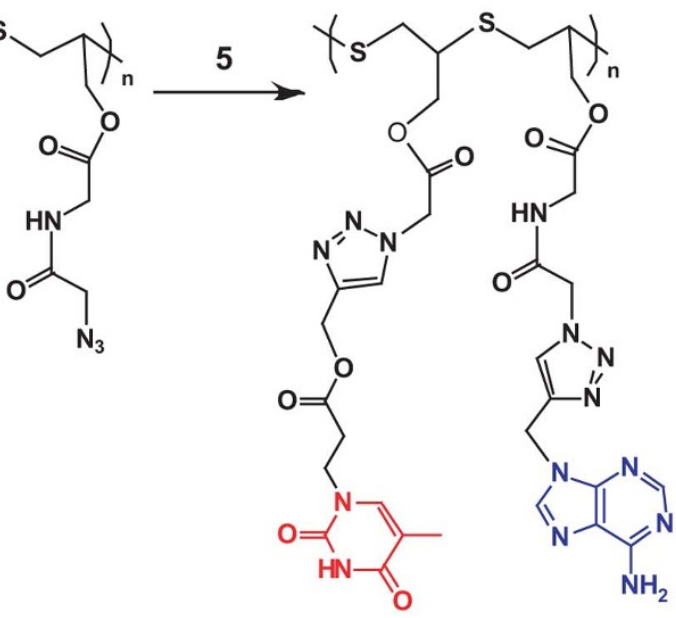

T6Thy-Y6Ade

Figure 4 Synthetic route to DNA-mimetic T6Thy-Y6Ade. The procedure includes: 1) esterifying-OH groups with azido-acetic acid in the presence of EDC and DMAP; 2) deprotection of Boc groups by 2, 2, 2-trifluoroacetic acid; 3) click coupling of $-\mathrm{N}_{3}$ groups with Thy-yne under CuBr and PMDETA; 4) amidation of $-\mathrm{NH}_{2}$ groups with azido-acetic acid in the presence of EDC and DMAP; 5) click coupling of - $\mathrm{N}_{3}$ groups with Ade-yne under CuBr and PMDETA. 
a

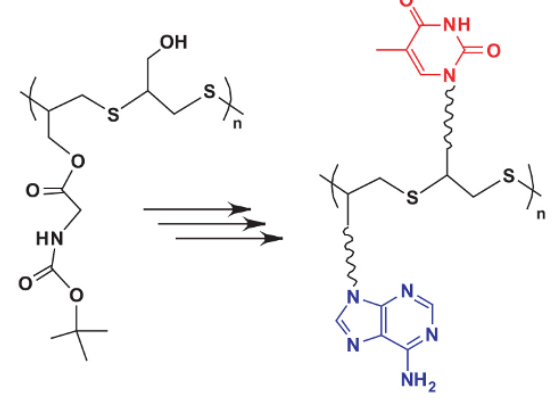

c

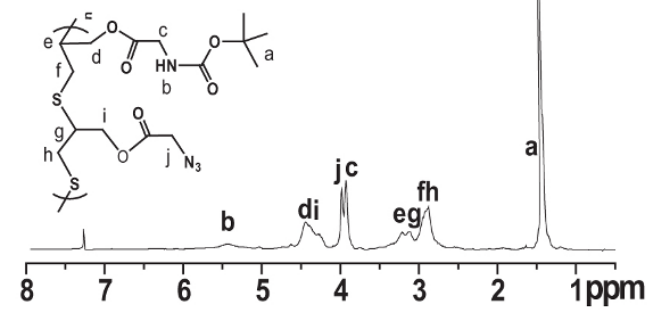

e

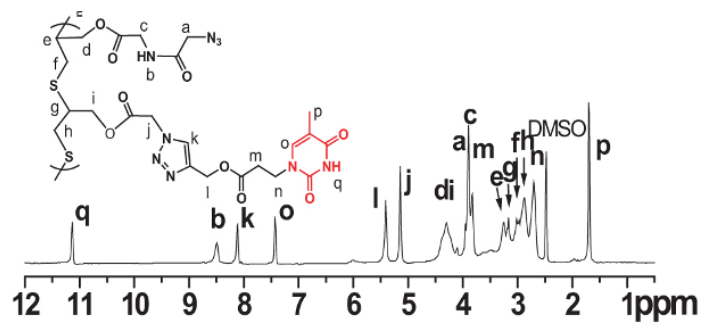

b

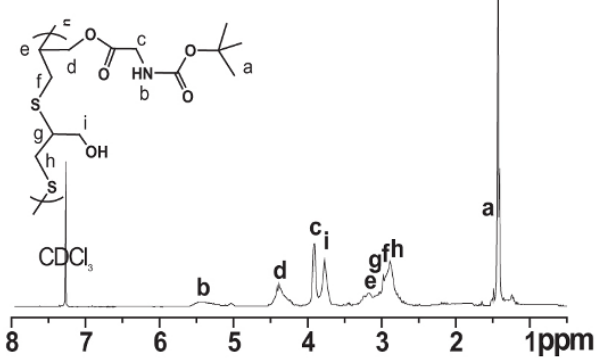

d

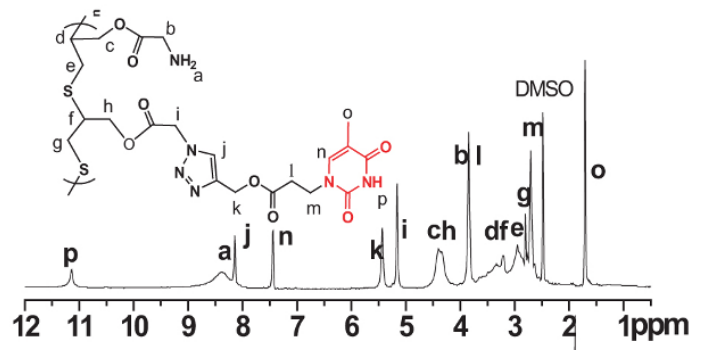

f

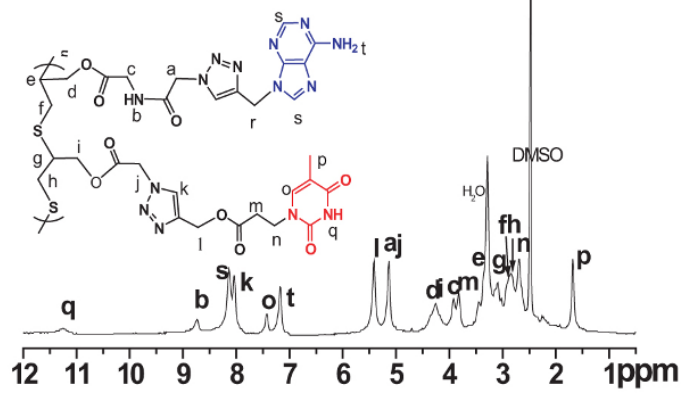

Figure 5 Characterization of DNA-mimetic polymers. (a)The process of T6-Y6 to T6Thy-Y6Ade; ${ }^{1} \mathrm{H}$ NMR spectra (b-f) of T6Thy-Y6Ade and intermediates.

(Y1-Y6). Series of novel polymers with alternatively arranged functional groups (e.g., -OH/-OH, -OH/-COOH, -OH/-alkyl, $\mathrm{OH} /-\mathrm{NH}_{2}$, etc.) were obtained by simple one-pot polymerization. Interestingly, more complex SCFPs with S-R1-R1-S-R2-type sequence were achieved by direct polymerization of $\mathrm{T} 5$ and alkynes (Y1-Y6). Five kinds of sequential polymers with high density of hetero-functional groups $\left(-\mathrm{CHOH} /-\mathrm{CHOH} /-\mathrm{CH}_{2} \mathrm{OH}\right.$, $\mathrm{CHOH} /-\mathrm{CHOH} /-\mathrm{SCH}_{2} \mathrm{OH},-\mathrm{CHOH} /-$

$\mathrm{CHOH} /-\mathrm{C}_{6} \mathrm{H}_{13},-\mathrm{CHOH} /-\mathrm{CHOH} /-\mathrm{COOH}$, and $-\mathrm{CHOH} /-\mathrm{CHOH} /-$ $\mathrm{NH}_{2}$, etc.) were also acquired in high yields $(>90 \%)$. Their structures were confirmed by the ${ }^{1} \mathrm{H}$ NMR and ${ }^{13} \mathrm{C}$ NMR spectra (Figures S13S20). The RISG polymerization strategy can easily offer such complex SCFPs with precisely hetero-functional sequence simply by selection of the appropriate monomers, which was never reported before.

Periodic and random SCFPs. Our strategy can be extended to synthesize SCFPs with more advanced topologies such as periodic, random, and hyperbranched polymers. To obtain periodic SCFPs, we designed the synthetic process in three steps (Figure $3 \mathrm{~b}$ ). First, $\alpha$, $\omega$-bifunctional prepolymer $\left(M_{\mathrm{n}}=1500 \mathrm{~g} \mathrm{~mol}^{-1}\right.$, PDI $\left.=1.80\right)$ was prepared by direct polymerization of T4 and Y1 for $2 \mathrm{~h}$. Second, a little excess of T4 was added into the reactive system ensuring the terminal groups to be thiols. Finally, the $\alpha, \omega$-dithiol oligomer with 9.4 average repeating units $\left(D P_{\mathrm{n}}=9.4\right)$ reacted with $N$,
$N^{\prime}$-methylene bisacrylamide in the presence of $\mathrm{Et}_{3} \mathrm{~N}$ to afford the targeted product of periodic SCFPs with S-R-S-R2-type sequence and $M_{\mathrm{n}}$ of $8600 \mathrm{~g} \mathrm{~mol}^{-1}\left(D P_{\mathrm{n}}=51.0\right.$, and PDI $\left.=2.1\right)$. Based on the average molecular weight, the backbone composed of $\sim 5 \alpha, \omega$ bifunctional oligomers and $\sim 4 N, N^{\prime}$-methylene bisacrylamide monomers. Additionally, the structure of SCFPs can also be tailored by changing the employed oligomers and the diacrylate chain extenders.

Furthermore, the random SCFP, T5-Y3-Y4, was prepared by copolymerization of hydrophilic T5 and Y4 with hydrophobic Y3. The structure of T5-Y3-Y4 was confirmed by ${ }^{1} \mathrm{H}$ NMR spectroscopy (Figure S21), and it has a $M_{\mathrm{n}}$ of $5700 \mathrm{~g} \mathrm{~mol}^{-1}$ with a relatively narrow PDI $\left(M_{\mathrm{w}} / M_{\mathrm{n}}=1.60\right)$. Since the reactivity of thiol-aliphatic 1-octyne (Y3) is higher than that of thiol-propargyl acetate (Y4) $)^{34-36}, \mathrm{Y} 3$ positioned within the chain is faster than Y4 as the reaction starts. So T5Y3-Y4 is an amphiphilic random SCFPs with gradient distribution of amphiphilicity.

Hyperbranched SCFPs. Hyperbranched polymers (HPs), widely expressed in biomacromolecules, are a main subclass of dendritic macromolecules with $3 \mathrm{D}$ architecture, promising wide range of applications from functional coatings and drug-carriers due to their unique properties such as high solubility, low viscosity and plenty of functional groups ${ }^{37-40}$. Unfortunately, most of artificial HPs possess only one kind of reactive groups in the periphery, 


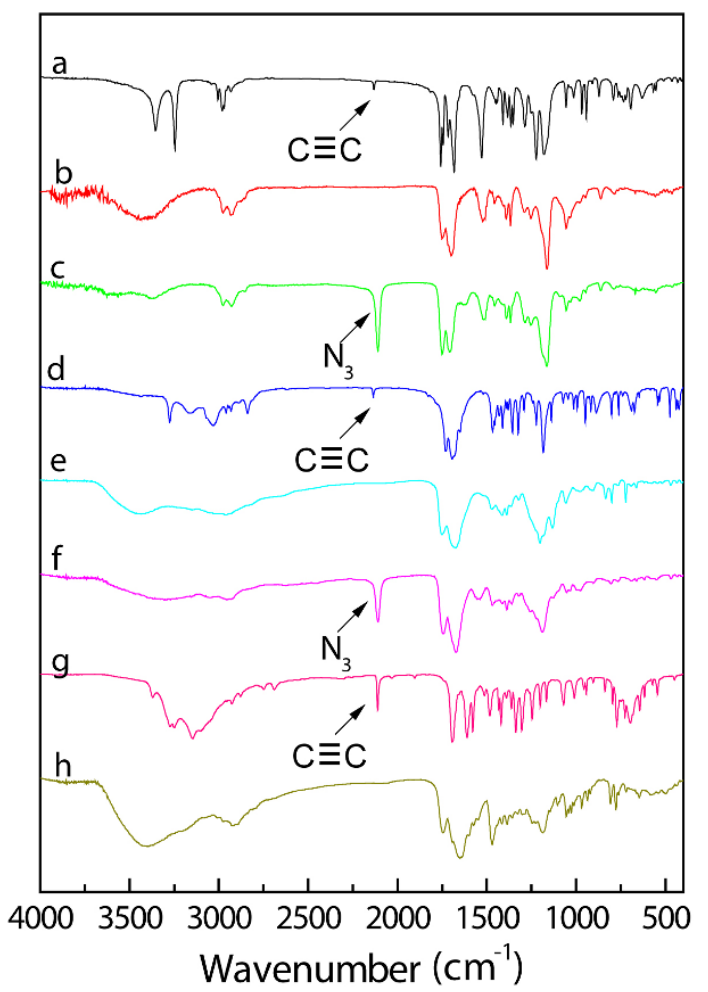

Figure 6 | FTIR spectra of T6Thy-Y6Ade and intermediates. (a) Y6, (b)

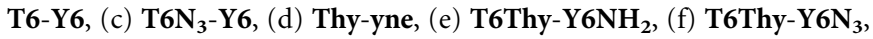
(g) Ade-yne, (h) T6Thy-Y6Ade.

limiting their selective functionalization. Recently, our group synthesized HPs with hetero-functional groups, but their sequences were uncontrollable ${ }^{41}$. Here we synthesized hyperbranched SCFPs via the RISG polymerization. To this aim, the monomer simultaneously containing both thiol and alkyne groups was synthesized at first. Typically, utilizing $\mathrm{KOH}$ as the catalyst, the reaction of $\mathrm{T} 5$ and propargyl bromide yielded $\mathrm{AB}_{2}$-type intermediate with S-R1OH-R2-R1OH-S sequence. Further polymerization of the $\mathrm{AB}_{2}$ species gave rise to S-R1OH-R2-R1OH-S-type hyperbranched SCFP $\left(M_{\mathrm{w}}=4800 \mathrm{~g} \mathrm{~mol}^{-1}\right.$ and PDI $\left.=1.27\right)$ (Fig. 2d). The corresponding ${ }^{1} \mathrm{H}$ NMR spectrum confirmed its dendritic structure and S-R1OH-R2-R1OH-S-type sequence (Figure S22). The hyperbranched SCFPs can be further modified selectively to develop novel materials, on the basis of pendent hetero-functional groups such as hydroxyl, thioether, and peripheral alkyne groups.

Artificial DNA with alternative adenine and thymine groups. To demonstrate the versatility of hetero-functional groups of SCFPs, we introduced alternating adenine (Ade) and thymine (Thy) groups on the side chain of linear SCFPs (e.g., T6-Y6 or T5-Y5) for achieving single-strand DNA-mimetic macromolecules. The synthetic route was displayed in Figure 3. The sequential polymer T6-Y6 $\left(M_{\mathrm{n}}=\right.$ $2400 \mathrm{~g} \mathrm{~mol}^{-1}$ and PDI $=1.42$ ) was firstly esterified with azidoacetic acid to afford $\mathrm{T}_{6} \mathrm{~N}_{3}-\mathrm{Y} 6$. Next, cleavage of Boc groups by treatment with trifluoroacetic acid yielded hygroscopic $\mathbf{T}_{6} \mathrm{~N}_{\mathbf{3}^{-}}$ $\mathrm{Y}_{6} \mathrm{NH}_{2}$ possessing alternative $-\mathrm{N}_{3} /-\mathrm{NH}_{2}$ groups. Subsequent reaction of azido groups of $\mathrm{T}^{6} \mathrm{~N}_{3}-\mathrm{Y}^{6} \mathrm{NH}_{2}$ and thy-yne via azidealkyne click coupling catalyzed by $\mathrm{CuBr} / \mathrm{PMDETA}$ afforded very hygroscopic T6Thy-Y6NH $\mathrm{NH}_{2}$. Amidation of T6Thy-Y6NH $\mathrm{N}_{2}$ with excess azido-acetic acid gave rise to T6Thy- $\mathrm{Y}^{6} \mathrm{~N}_{3}$ with the complete conversion of $\mathrm{NH}_{2}$ into azides. Finally, click coupling of ade-yne to T6Thy-Y6N 3 gave birth to DNA-mimetic SCFP, T6ThyY6Ade, with sequential adenine and thymine functional moieties
(Figure 4). The accumulated $\mathrm{Cu}$ (I) catalyst was removed by twice precipitating out the polymer from ethylene diamine tetraacetic acid (EDTA) saturated aqueous solution. The whole synthesis steps were monitored by ${ }^{1} \mathrm{H}$ NMR spectroscopy (Figure 5) and Fourier transform infrared (FTIR) spectra (Figure 6), confirming that Thy and Ade units were alternatively introduced onto the SCFP successfully with the complete consuming of azide groups of polymers. Similar to DNA, the artificial DNA macromolecules can also self-assemble into micelles by self-recognition of Ade and Thy base-pair, as confirmed by dynamic light scattering (DLS) and atomic force microscopy (AFM) (Figure S23, S24).

\section{Discussion}

We designed and implemented RISG polymerization protocol capable of achieving diverse SCFPs that have no necessary specific mental-catalyst and do not need tedious protection and deprotection process. Notably, the large chain transfer constant $(>48)$ of thiol groups is the key factor to obtain the SCFPs ${ }^{31}$. As shown in Figure 1, all the carbon-centered radicals are quenched by thiolterminated molecules, avoiding homopolymerizations of alkynyl monomers. Moreover, the living radicals have no chance to carry out the hydrogen abstraction from functional groups (such as hydroxyl and carboxyl groups, etc.) which are intact after the stepgrowth reaction. In contrast, for the traditional condensation polymerization, except the terminal groups, most of the functional ones would be consumed during the chain extension. Therefore, the resulted SCFPs are ascribed to the step-growth polymerization triggered by radicals.

Molecular weight is an important parameter to macromolecules. Here, we regulate the molecular weight of the SCFPs mainly by controlling the conversion and the feeding ratio of thiol and alkyne groups. With prolonging the reaction time, the molecular weight of SCFPs increases similar to the chain extension fashion involved in traditional polycondensation (Table S1). As the conversion reaches the predetermined level, the propagation will be terminated at once by decreasing the reactive temperature. In this work, we fixed the feeding ratio of thiol and alkynyl groups to be about $1: 1$ (thiol groups are slightly excess), ensuring the resulted SCFPs be of higher molecular weight and the thiol-ended groups (Figure 1). These thiol-capped SCFPs can further react with diacrylates to present periodic ones (Figure 3b).

Based on the mechanism of RISG polymerization, thiyl radicals successively perform twice addition reactions on the same alkyne monomer. It has been reported that, during the thiol-ene addition reaction, such side reactions as the thiyl-thiyl and the carbon center radical coupling have been observed ${ }^{42,43}$. In this work, the GPC profile of T1-Y1 prepared in bulk is bimodal (Figure 2a), indicating the existence of macroradicals coupling. In bulk polymerization, the high radical concentration and the large viscosity increase the coupling probability. In contrast, carrying out the RISG polymerization of T1Y1 in water or solutions, the corresponding GPC profiles are unimodal, implying such coupling reactions as shown in Figure 7 can be greatly avoided.

As mentioned above, our strategy gives fully expression to the flexibility of topological design of SCFPs. The multiple topologies such as, linear, periodic, random and hyperbranched architectures are feasible to achieve only by changing the employed monomers. For instance, the complex hyperbranched SCFP was successfully synthesized using $\mathrm{AB}_{2}$-type monomer via the RISG polymerization (Figure 3d). It is quite difficult to synthesize SCPs for those reported methods without the helps of other synthetic strategies since their monomers or catalysts are special and specific. Therefore, our strategy is an efficient tool to design the molecular structures of SCFPs.

Compared to the traditional condensation polymerization, there are no small molecules released and no chemical reaction balance existed during the RISG process, displaying its atom economy and 
(S-1)

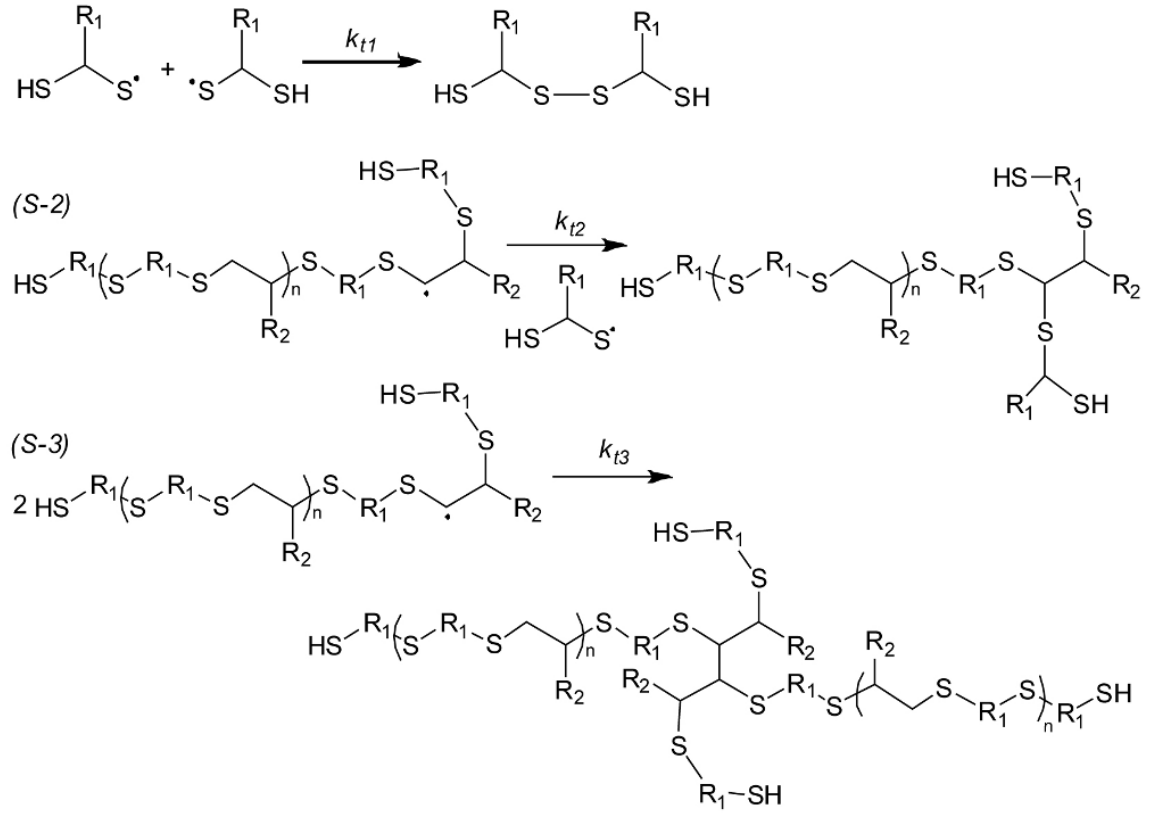

Figure $7 \mid$ Possible side reactions involved in the RISG polymerization.

high efficiency. Additionally, it is not necessary to perform experiments under those rigorous synthesis conditions. For instance, water is a strict limit during most of the previous polymerization procedures because the employed metal-catalysts and the active centers are humidity-sensitive $e^{9,13,14,29,30}$. By comparison, following the different mechanism (Figures 1a and 2b), our RISG polymerization proceeds smoothly without metal-catalyst and thus offered the targeted products in water. These results also demonstrated that the RISG polymerization protocol is facile and robust indeed to access SCPs with independently functional groups, which can be further used to synthesize more complex biomimetic materials.

At this stage, the DNA-mimetic polymers with alternative Adenine and Thymine side groups were synthesized via the RISG polymerization (Figure 4). This strategy can be expanded to give birth to SCPs possessing Guanine and Cytosine bases, contributing to fully understand the self-assembled behaviors of DNA strands.

In conclusion, we presented a general avenue to synthesize tailormade hetero-functional sequences with a wide variety of topologies. This strategy has many merits: needless protection process, fast reaction, precise sequence, and high yield. The independently functional groups along the main chain were converted into adenine and thymine units, offering artificial DNA macromolecules. The dithiol or alkyne monomers can be flexibly synthesized as required, which provides a perfect platform for facile synthesis of SCFPs. Our work declares the feasibility of artificial sequences with advanced functions similar to DNA self-recognition, opening a door to complex molecular design for artificial life.

\section{Methods}

For complete experimental methods see Supplementary Information.

Synthesis of linear SCFP T1-Y1. Typically, T1 $(1.82 \mathrm{~g}, 10 \mathrm{mmol})$ and Y1 $(0.56 \mathrm{~g}$, $10 \mathrm{mmol}$ ) were mixed with $2 \mathrm{~mL}$ of distilled water containing ACVA $(56.0 \mathrm{mg}$, $0.20 \mathrm{mmol}$ ) in a $25 \mathrm{~mL}$ Schlenk flask. $\mathrm{N}_{2}$ was purged to eliminate oxygen. The flask was then placed in an oil bath thermostated at $70^{\circ} \mathrm{C}$. After polymerization for $7 \mathrm{~h}$, the mixture was removed water by rotary evaporator, redissolved by tetrahydrofuran, and then poured into cold petroleum ether to precipitate out the polymers. Repeated dissolution, precipitation and subsequent vacuum drying afforded $2.26 \mathrm{~g}$ of the polymer with a yield of $95 \%$.

It should be noted that, except Y4 (in DMF), the RISG polymerization smoothly proceeded in water between all the dithiols (T1-T7) and alkynes (Y1-Y7) to achieve the SCFPs, because Y4 was acidic in water and tended to occur in hydrolysis by self-catalysis. Notably, all the RISG polymerization mentioned above can be triggered by $3 \%$ DMPA under UV-radiation in bulk or in solutions.

Instruments. ${ }^{1} \mathrm{H}$ and ${ }^{13} \mathrm{C}$ nuclear magnetic resonance (NMR) spectra were acquired on a Varian Mercury Plus $400 \mathrm{MHz}$ spectrometer at $20^{\circ} \mathrm{C}$. Molecular weights and molecular weight distributions were determined by gel permeation chromatography (GPC) using PE series 200 with RI-WAT 150CV+ as detector, THF as the mobile phase at a flow rate of $1 \mathrm{~mL} / \mathrm{min}$ and polyethylene glycol (PEG) as a standard at $70^{\circ} \mathrm{C}$. Light scattering data were recorded through Viscotek/Malvern GPC system consisting of a GPC Max autoinjector fitted to a TDA 305 triple detector array (differential RI, right angle light scattering (RALS), low angle static light scattering (LALS), and four-capillary differential viscometer detectors) using LiBr/DMF $(0.02 \mathrm{~mol} / \mathrm{L})$ as eluent at a flow rate of $0.7 \mathrm{~mL} / \mathrm{min}$ and linear poly $($ methyl methacrylate) as calibration at $50^{\circ} \mathrm{C}$. Fourier transform infrared (FTIR) spectra were recorded on a PE Paragon 1000 spectrometer ( $\mathrm{KBr}$ disk).

1. Lutz, J.-F., Ouchi, M., Liu, D. R. \& Sawamoto, M. Sequence-controlled polymers. Science 341, 1238149 (2013).

2. Aldaye, F. A., Palmer, A. L. \& Sleiman, H. F. Assembling materials with DNA as the guide. Science 321, 1795-1799 (2008).

3. Inaki, Y. Synthetic nucleic acid analogs. Prog. Polym. Sci. 17, 515-570 (1992).

4. Smith Jr, W. T. Nucleic acid models. Prog. Polym. Sci. 21, 209-253 (1996).

5. Lutz, J.-F. \& Börner, H. G. Modern trends in polymer bioconjugates design. Prog. Polym. Sci. 33, 1-39 (2008).

6. Ueda, M. Sequence controlled in one-step condensation polymerization. Prog. Polym. Sci. 24, 699-730 (1999).

7. Hibi, Y., Tokuoka, S., Terashima, T., Ouchi, M. \& Sawamoto, M. Design of AB divinyl "template monomers" toward alternating sequence control in metalcatalyzed living radical polymerization. Polym. Chem. 2, 341-347 (2011).

8. Merrifield, R. B. Solid phase peptide synthesis. I. The synthesis of a tetrapeptide. J. Am. Chem. Soc. 85, 2149-2154 (1963).

9. Kramer, J. W. et al. Polymerization of enantiopure monomers using syndiospecific catalysts: A new approach to sequence control in polymer synthesis. J. Am. Chem. Soc. 131, 16042-16044 (2009).

10. Lutz, J.-F., Schmidt, B. V. K. J. \& Pfeifer, S. Tailored polymer microstructures prepared by atom transfer radical copolymerization of styrene and $\mathrm{N}$-substituted maleimides. Macromol. Rapid Commun. 32, 127-135 (2011).

11. Satoh, K., Matsuda, M., Nagai, K. \& Kamigaito, M. AAB-sequence living radical chain copolymerization of naturally occurring limonene with maleimide: An endto-end sequence-regulated copolymer. J. Am. Chem. Soc. 132, 10003-10005 (2010).

12. Satoh, K., Ozawa, S., Mizutani, M., Nagai, K. \& Kamigaito, M. Sequence-regulated vinyl copolymers by metal-catalysed step-growth radical polymerization. Nat. Commun. 1, 6-11 (2010)

13. Song, A., Parker, K. A. \& Sampson, N. S. Synthesis of copolymers by alternating ROMP (AROMP). J. Am. Chem. Soc. 131, 3444-3445 (2009). 
14. Robert, C., Montigny, F. \& Thomas, C. M. Tandem synthesis of alternating polyesters from renewable resources. Nat. Commun. 2, 256-261 (2011).

15. Badi, N. \& Lutz, J.-F. Sequence control in polymer synthesis. Chem. Soc. Rev. 38, 3383-3390 (2009).

16. Lutz, J.-F. A controlled sequence of events. Nat. Chem. 2, 84-85 (2010).

17. McHale, R. \& O’Reilly, R. K. Nucleobase containing synthetic polymers: Advancing biomimicry via controlled synthesis and self-assembly. Macromolecules 45, 7665-7675 (2012).

18. Safak, M., Alemdaroglu, F. E., Li, Y., Ergen, E. \& Herrmann, A. Polymerase chain reaction as an efficient tool for the preparation of block copolymers. Adv. Mater. 19, 1499-1505 (2007).

19. Uhlmann, E., Peyman, A., Breipohl, G. \& Will, D. W. PNA: synthetic polyamide nucleic acids with unusual binding properties. Angew. Chem., Int. Ed. 37, 2796-2823 (1998).

20. Rose, K. \& Vizzavona, J. Stepwise solid-phase synthesis of polyamides as linkers. I. Am. Chem. Soc. 121, 7034-7038 (1999).

21. Li, X. \& Liu, D. R. DNA-templated organic synthesis: nature's strategy for controlling chemical reactivity applied to synthetic molecules. Angew. Chem., Int. Ed. 43, 4848-4870 (2004).

22. Rojas Stütz, J. A. \& Richert, C. A steroid cap adjusts the selectivity and accelerates the rates of nonenzymatic single nucleotide extensions of an oligonucleotide. J. Am. Chem. Soc. 123, 12718-12719 (2001).

23. Rosenbaum, D. M. \& Liu, D. R. Efficient and sequence-specific DNA-templated polymerization of peptide nucleic acid aldehydes. J. Am. Chem. Soc. 125 13924-13925 (2003)

24. Li, X., Zhan, Z. Y. J., Knipe, R. \& Lynn, D. G. DNA-catalyzed polymerization. J. Am. Chem. Soc. 124, 746-747 (2002).

25. Kleiner, R. E., Brudno, Y., Birnbaum, M. E. \& Liu, D. R. DNA-templated polymerization of side-chain-functionalized peptide nucleic acid aldehydes. J. Am. Chem. Soc. 130, 4646-4659 (2008).

26. Datta, B., Schuster, G. B., McCook, A., Harvey, S. C. \& Zakrzewska, K. DNAdirected assembly of polyanilines: Modified cytosine nucleotides transfer sequence programmability to a conjoined polymer. J. Am. Chem. Soc. 128 , 14428-14429 (2006)

27. Datta, B. \& Schuster, G. B. DNA-directed synthesis of aniline and 4aminobiphenyl oligomers: Programmed transfer of sequence information to a conjoined polymer nanowire. J. Am. Chem. Soc. 130, 2965-2973 (2008)

28. Walde, P. \& Guo, Z. Enzyme-catalyzed chemical structure-controlling template polymerization. Soft Matter 7, 316-331 (2011).

29. Leclerc, M. K. \& Waymouth, R. M. Alternating ethene/propene copolymerization with a metallocene catalyst. Angew. Chem., Int. Ed. 37, 922-925 (1998).

30. Choi, T.-L., Rutenberg, I. M. \& Grubbs, R. H. Synthesis of A, B-alternating copolymers by ring-opening-insertion-metathesis polymerization. Angew. Chem., Int. Ed. 41, 3839-3841 (2002).

31. Pan, Z. R. Polymer Chemistry (Chemical Industry, Beijing, 2011).

32. Hibi, Y., Tokuoka, S., Terashima, T., Ouchi, M. \& Sawamoto, M. Design of AB divinyl "template monomers" toward alternating sequence control in metalcatalyzed living radical polymerization. Polym. Chem. 2, 341-347 (2011).

33. Hibi, Y., Ouchi, M. \& Sawamoto, M. Sequence-regulated radical polymerization with a metal-templated monomer: repetitive $\mathrm{ABA}$ sequence by double cyclopolymerization. Angew. Chem., Int. Ed. 50, 7434-7437 (2011).

34. Fairbanks, B. D., Sims, E. A., Anseth, K. S. \& Bowman, C. N. Reaction rates and mechanisms for radical, photoinitated addition of thiols to alkynes, and implications for thiol-yne photopolymerizations and click reactions. Macromolecules 43, 4113-4119 (2010).
35. Chan, J. W., Hoyle, C. E. \& Lowe, A. B. Sequential phosphine-catalyzed, nucleophilic thiol-ene/radical-mediated thiol-yne reactions and the facile orthogonal synthesis of polyfunctional materials. J. Am. Chem. Soc. 131, 5751-5753 (2009).

36. Fairbanks, B. D., Scott, T. F., Kloxin, C. J., Anseth, K. S. \& Bowman, C. N. Thiolyne photopolymerizations: Novel mechanism, kinetics, and step-growth formation of highly cross-linked networks. Macromolecules 42, 211-217 (2009).

37. Gao, C. \& Yan, D. Y. Hyperbranched polymers: from synthesis to applications. Prog. Polym. Sci. 29, 183-275 (2004).

38. Kim, Y. \& Webster, O. Water soluble hyperbranched polyphenylene: “a unimolecular micelle?" J. Am. Chem. Soc. 112, 4592-4593 (1990).

39. Jikei, M. \& Kakimoto, M. Hyperbranched polymers: a promising new class of materials. Prog. Polym. Sci. 26, 1233-1285 (2001).

40. Yan, D. Y., Gao, C. \& Frey, H. Hyperbranched Polymers: Synthesis, Properties, and Applications (Wiley, New York, 2011).

41. Han, J., Zhao, B., Tang, A. J., Gao, Y. Q. \& Gao, C. Fast and scalable production of hyperbranched polythioether-ynes by a combination of thiol-halogen click-like coupling and thiol-yne click polymerization. Polym. Chem. 3, 1918-1925 (2012)

42. Chan, J., Yu, B., Hoyle, C. E. \& Lowe, A. B. The nucleophilic, phosphine-catalyzed thiol-ene click reaction and convergent star synthesis with RAFT-prepared homopolymers. Polymer 50, 3158-3168 (2009).

43. Koo, S. et al. Limitations of radical thiol-ene reactions for polymer-polymer conjugation. J. Polym. Sci.: Part A: Polym. Chem. 48, 1699-1713 (2010).

\section{Acknowledgments}

We acknowledge the financial supports from the National Natural Science Foundation of China (No. 51173162 and No. 21325417), Qianjiang Talent Foundation of Zhejiang Province (2010R10021), the Fundamental Research Funds for the Central Universities (2013XZZX003), China Postdoctoral Science Foundation (No. 20100471707 and No. 201104716), and Zhejiang Provincial Natural Science Foundation of China (No. R4110175).

\section{Author contributions}

C.G. conceived the concept, C.G., J.H. and Y. Zheng designed the research, analyzed the experimental data and prepared the manuscript; B.Z., S.L. and Y. Zhang conducted part of experiments; C.G. supervised and directed the project; all the authors read and revised the paper.

\section{Additional information}

Supplementary information accompanies this paper at http://www.nature.com/ scientificreports

Competing financial interests: The authors declare no competing financial interests.

How to cite this article: Han, J. et al. Sequentially Hetero-functional, Topological Polymers by Step-growth Thiol-yne Approach. Sci. Rep. 4, 4387; DOI:10.1038/srep04387 (2014).

This work is licensed under a Creative Commons AttributionNonCommercial-NoDerivs 3.0 Unported license. To view a copy of this license, visit http://creativecommons.org/licenses/by-nc-nd/3.0 